\title{
PDI-mediated mesenchymal stem cells immunemodulation: the two sides of the coin
}

\begin{abstract}
Mesenchymal Stem Cells (MSC) can be considered as part of the immune system, taking an active role in regulating both the innate and adaptive response by the combined action of secreted factors and membrane receptors. Among the different inhibitory receptors shown to be involved in the MSC-mediated immumoregulation, the Programmed Death 1 (PD1) pathway is in the unique position of being also a major component of the immunosuppressive tumor microenvironment and a target for cancer immunotherapy. On these premises, here we discuss how PD1-mediated MSC immunesuppression could be beneficial treating immune-mediated conditions or detrimental when exerted in the tumor microenvironment.
\end{abstract}

Keywords: Mesenchymal Stem Cells, PD1 pathway, PD1 blockade, immunemodulation, immune-mediated diseases, tumor microenvironment.
Volume 6 Issue 4 - 2018

\author{
Giuseppina Pennesi \\ Area Vasta 4 Fermo-ASUR Marche, Italy
}

Correspondence: Giuseppina Pennesi, Area Vasta 4 FermoASUR Marche,Via Zeppilli I8, Fermo, Italy, Tel +39-338 829I259, Email pinapennesi@hotmail.com

Received: May 0I, 2018 | Published: August 10,2018
Abbreviations: MSC, mesenchymal stem cells; NK natural killer; GHVD, graft versus host disease, TGF $\beta$, transforming growth factor $\beta$; HGF, hepatocyte growth factor; IDO, Indole amine 2,3-dioxygenase; NO, nitric oxide ; HLA G5, Human Leukocyte Antigen G5; $\mathrm{PGE}_{2}$, Prostaglandin $\mathrm{E}_{2}$; IL10, Interleukin 10; VCAM1 and ICAM1, Vascular and Intercellular Cell Adeshion Molecule 1; TCR, T Cell Receptor

\section{Mesenchymal stem cells}

According to the Mesenchymal and Tissue Stem Cell Committee of the International Society for Cellular Therapy, Mesenchymal Stem Cells (MSC) are defined as multipotent mesenchymal cells that can be found in several disparate tissues and can differentiate into osteoblasts, adipocytes, and chondroblasts, under appropriate culture conditions. ${ }^{1,2}$ The first evidences that MSC can have immunomodulatory properties were published more than a decade ago. ${ }^{3,4}$ followed by the first successful report of cell therapy with MSC for Graft Versus Host Disease (GVHD) in human. ${ }^{5}$ Within the Immune System, MSC act on the innate response by hampering the proliferation and activation of macrophages and Natural Killer (NK) cells, and on the adaptive immunity blocking the proliferation and activation of $\mathrm{T}$ and $\mathrm{B}$ lynphocytes. ${ }^{3,4}$ MSC immunosuppressant function is due to the redundant combination of secreted factors and membrane receptors engaged in cell-to-cell contact. Activated MSC produce several inhibitory cytokines, such as Transforming Growth Factor $\beta$ (TGF $\beta$ ), Hepatocyte Growth Factor (HGF), Indole amine 2,3-dioxygenase (IDO), Nitric Oxide (NO), Human Leukocyte Antigen G5 (HLA G5), Prostaglandin $\mathrm{E}_{2}\left(\mathrm{PGE}_{2}\right)$, and Interleukin 10 (IL10). They express also the inhibitory receptors Cytotoxic T-Lymphocyte Antigen 4 (CTLA4), HLA-G1, Vascular and Intercellular Cell Adeshion Molecule 1 (VCAM1 and ICAM1), and PD1/PD-Ligand 1 (PD-L1).,

\section{PD I-mediated MSC immunesuppression}

PD1 is expressed on activated T and B cells and its engagement by its ligands PD-L1 or PD-Ligand 2 (PD-L2) disrupts kinase activity in the T Cell Receptor (TCR)-activation cascade. The resulting immunosuppresion involves T-cell apoptosis, T-cell exhaustion, T-cell anergy, T-cell IL10 production, and T regulatory cell (Treg) induction. ${ }^{6}$ To exert their immunomodulant activity, MSC need to be embedded in an activated milieu, such as in presence of an anti-self response, a chronic viral infection, or in a tumor microenvironment. Under these conditions, pro-inflammatory and suppressive factors interact in a balanced loop. In particular, Interferon $\gamma$ (IFN $\gamma$ ) increases the expression of PD-L1 on MSC, ${ }^{7}$ and the cognate contact between PD-L1-espressing MSC and the PD1-expressing lymphocytes activate an immunosuppressive response. ${ }^{8}$ On the other hand, some data show that the engagement between PD-L1 on MSC and PD1 on T lymphocytes leads to the generation of FOXP3 + Treg, contributing to the blockade of T cell activation. ${ }^{6}$

\section{Two settings, different outcomes:} immune-mediated conditions and tumor microenvironment

Thus far, more than six hundred clinical trials are listed in the www.ClinicalTrials.gov web site using MSC as a pharmaceutical tool for tissue regenerative or immunosuppressive purposes. What makes MSC attractive for cell therapy is their ability to home to the sites of inflammation and injury releasing growth factors or cytokines to promote tissue repair or to regulate the activation of inflammation and immune responses. Their use as cell therapy for immune-mediated diseases has been explored in pre-clinical and clinical settings. They attenuate signs and symptoms in mouse models of immune-mediated diseases, the efficacy of MSC therapy has been initially described in Collagen-Induced Arthritis, ${ }^{9}$ later in Experimental Autoimmune Uveitis, ${ }^{10}$ and in many others. ${ }^{11}$ In humans, results have been encouraging for some conditions, such as Crohn's disease and Multiple Sclerosis, but not so conclusive in others, such as GVHD, Systemic Lupus Erythematosus, and Rheumatoid Arthritis, ${ }^{12}$ possibly due to differences in disease-specific pathogenic mechanisms or to therapeutic protocols non concordant in terms of cell sources, in vitro expansion protocols, routes and schedules of administration. In this sense, other approaches are currently tested, eventually skewing the problems associated with donor variability and ex vivo cell expansion, i.e. the use of MSC extracellular vescicles. ${ }^{13}$

A burden on the clinical use of MSC is their oncogenic potential, 
although the published data are controversial. It has been shown in in vivo and ex vivo settings that MSC can develop into sarcoma, ${ }^{14}$ and favor tumor growth by preventing tumor recognition by the immune system $;{ }^{15}$ they also induce the metastasis of breast cancer cells constituting a stroma delivering a continuous tumorigenic stimulus for metastasis of breast cancer cells. ${ }^{16}$ Multiple other studies, however, have demonstrated that human MSC are resistant to spontaneous transformation, and a more careful revision of clinical trials data didn't show any association between MSC treatment and cancer development. ${ }^{17}$ We need, however, to distinguish between the oncogenic potential of infused or implanted MSC after ex vivo expansion, which might be due to manipulation or to cell contamination, and the tumorigenic potential of MSC embedded in the tumor niche. The immunosuppressant function of MSC in the tumor microenvironment can be considered detrimental in terms of tumor growth. In fact, MSC can dampen any early innate response of the host to tumor growth and T-cell reaction to transformed cells. ${ }^{18}$ In this context, the role of the PD1/PD-L1 pathway can be pivotal, for that the induction of PD1 or PD-L1 expression on MSC decreases the cytotoxic effect of tumor specific T cells. ${ }^{19}$ Moreover, the therapeutic effect observed upon blockade of PD1/PD-L1 with monoclonal antibodies in solid tumors can be due to the direct effect on the interaction between tumor cell and effector lymphocyte, but also to the shut down of the inhibiting signal elicited by the PD1/PDL1 binding during contact between effector lymphocyte and MSC. ${ }^{19}$ Immunotherapy is aimed at boosting the natural defense against cancer, and the use of PD1/PD-L1 checkpoint inhibitors increases the activity of self-reacting T-cells, by inhibiting the induction of Treg and activating $\mathrm{T}$ effector cells. The contrasting scenario is exuberant immunity, leading to immune-mediated tissue injury and autoimmune diseases..$^{20}$ Indeed, a systematic review and meta analysis of literature on anti-PD1 drugs showed that, although organ-specific immunerelated adverse events are uncommon, the risk is increased compared with control treatments. ${ }^{21}$

\section{Acknowledgements}

None.

\section{Conflicts of interest}

The author declares that there is no conflict of interest.

\section{References}

1. Horwitz EM, Le Blanc K, Dominici M, et al. Clarification of the nomenclature for MSC. The International Society for Cellular Therapy position statement. Cytotherapy. 2005;7(5):393-395.

2. Dominici M, Le Blanc K, Mueller I, et al. Minimal criteria for defining multipotent mesenchymal stromal cells. The International Society for Cellular Therapy position statement. Cytotherapy. 2006;8(5):315-317.

3. Tasso R, Pennesi G. When stem cells meet immunoregulation International Immunopharmacology. 2009;9(5):596-598.

4. Castro Manrreza ME, Montesinos JJ. Immunoregulation by Mesenchymal Stem Cells: Biological Aspects and Clinical Applications. J Immunol Res. 2015;394917.
5. Le Blanc K, Rasmusson I, Sundberg B, et al. Treatment of severe acute graft-versus-host disease with third party haploiden- tical mesenchymal stem cells. Lancet. 2004;363(9419):1439-1441.

6. Sharpe AH, Pauken KE. The diverse functions of the PD1 inhibitory pathway. Nat Rev Immunol. 2018;18(3):153-167.

7. Sheng H, Wang Y, Jin Y, et al. A critical role of IFN $\gamma$ in priming MSCmediated suppression of $\mathrm{T}$ cell proliferation through up-regulation of B7-H1. Cell Research. 2008;18(8):846-857.

8. Augello A, Tasso R, Negrini SM, et al. Bone Marrow Mesenchymal progenitor cells inhibit lymphocyte proliferation by activation of the programmed death 1 pathway. Eur J Immunol. 2005;35(5):1482-1490.

9. Augello A, Tasso R, Negrini SM, et al. Cell Therapy Using Allogeneic Bone Marrow Mesenchymal Stem Cells Prevents Tissue Damage in Collagen-Induced Arthritis. Arthritis Rheum. 2007;56(4):1175-1186.

10. Tasso R, Ilengo C, Quarto R, et al. Mesenchymal Stem Cells Induce Functionally Active T-Regulatory Lymphocytes in a Paracrine Fashion and Ameliorate Experimental Autoimmune Uveitis. Invest Opht Vis Sci. 2012;53(2):786-793

11. Klinker MW, Wei CH. Mesenchymal stem cells in the treatment of inflammatory and autoimmune diseases in experimental animal models. World J Stem Cells. 2015;7(3):556-567.

12. Pistoia V, Raffaghello L. Mesenchymal stromal cells and autoimmunity. Int Immunol. 2017;29(2):49-58.

13. Sharma J, Hampton JM, Valiente GL. Therapeutic development of Mesenchymal stem cells or their extracellular vescicles to inhibit autoimmune-mediated inflammatory processes in systemic lupus erythematosus. Front Immunol. 2017;8:526.

14. Tasso R, Augello A, Caridà M, et al. Development of sarcomas in mice implanted with mesenchymal stem cells seeded onto bioscaffolds. Carcinogenesis. 2009;30(1):150-157.

15. Ramasamy R, Lam EW, Soiero I, et al. Mesenchymal stem cells inhibit proliferation and apoptosis of tumor cells: impact on in vivo tumor growth. Leukemia. 2007;21(2):304-310.

16. Karnoub AE, Dash AB, Vo AP, et al. Mesenchymal stem cells within tumour stroma promote breast cancer metastasis. Nature. 2007;449(7162):557-563.

17. Casiraghi F, Remuzzi G, Abbate M, et al. Multipotent mesenchymal stromal cell therapy and risk of malignancies. Stem Cell Review. 2013;9(1):65-79.

18. Omalley G, Heijltjes, Houston AM, et al. Mesenchymal stromal cells (MSCs) and colorectal cancer: a troublesome twosome for the antitumor immune response? Oncotarget. 2016;7(37):60752-60770.

19. Poggi A, Varesano S, Zocchi R. How to hit mesenchymal stromal cells and make the tumor microenvironment immunostimulant rather than immunosuppressive. Front Immunol. 2018;9:262.

20. Zhang H, Watanabe R, Berry GJ. Immunoinhibitory checkpoint deficiency in medium and large vessel vasculitis. Proc Natl Acad USA. 2017;114(6):E970-E979.

21. Baxi S, Yang A, Gennarelli RL, et al. Immune-related adverse events for anti-PD1 and anti-PD-L1 drugs: systematic review and meta-analysis. BMJ. 2018;360:k793. 